\title{
The Radio Jet Velocities at High Resolution
}

\author{
Gabriele Giovannini \\ Dipartimento di Astronomia, Universita' di Bologna, via Ranzani 1, 40127 Bologna \\ and Istituto di Radioastronomia del CNR, via Gobetti 101, 40129 Bologna (Italy)
}

\begin{abstract}
The different methods to derive the jet velocity and orientation on the parsec scale are presented. From these methods I will discuss the velocity distribution of parsec scale jets and the possible presence of acceleration or deceleration in the jet velocity. Moreover, evidences of jet velocity structures will be reported. Finally I will present new data on the superluminal giant radio source $1144+35$ to discuss in detail the properties of pc scale jets.
\end{abstract}

Key words: AGN, radio jets, jet velocity

\section{Introduction}

Jets are the structures through which energy and matter can flow from the Active Galactic Nucleus (AGN) to the extended radio lobes. There are now widely accepted lines of evidences for the existence of relativistic bulk velocities in the parsec scale jets of radio sources. This result implies that the observational data are affected by relativistic effects (Doppler boosting) and provides the basis of the current unified theories (see e.g. (18)), which suggest that the appearance of an AGN strongly depends on the orientation. However, it is not well understood if the jet velocity is

Email address:

ggiovann@ira.cnr.it (Gabriele

Giovannini).

Preprint submitted to Elsevier Science the same in all radio sources, if it is correlated to other source properties as the radio power, and if velocity structures are common.

In this review I will discuss the jet kinematic properties.

\section{The Measurement of the Jet Velocity}

\subsection{Proper Motion}

Many AGNs contain compact radio sources with different components which appear to move apart. Multi epoch studies of these sources allow a direct measure of the apparent jet pattern velocity $\left(\beta_{a}\right)$. The observed distribution of the apparent velocity 
shows a large range of values (19); (12). From the measure of $\beta_{a}$ we can derive constraints on $\beta_{p}$ and $\theta$ where $\beta_{p}$ is the intrinsic velocity of the pattern flow and $\theta$ is the jet orientation with respect to the line of sight:

$\beta_{p}=\beta_{a} /\left(\beta_{a} \cos \theta+\sin \theta\right)$

A main problem is to understand the difference between the bulk and pattern velocity. In few cases where proper motion is well defined and the bulk velocity is strongly constrained, there is a general agreement between the pattern velocity and the bulk velocity (see e.g. NGC 315 , (5), and 1144+35, here). However, in the same source we can have different pattern velocities as well as stationary and high velocity moving structures. Moreover, we note that in many well studied sources the jet shows a smooth and uniform surface brightness and no (or very small) proper motion (as in the case of Mkn 501, Giroletti et al. in preparation, and M87 in the region at $\sim 1 \mathrm{pc}$ from the core (14)).

\subsection{Jet Sidedness}

Assuming that the jets are intrinsically symmetric we can use the observed jet to counter-jet brightness ratio $\mathrm{R}$ to constrain the jet bulk velocity $\beta c$ and its orientation with respect to the line of sight $(\theta)$ :

$\mathrm{R}=(1+\beta \cos \theta)^{2+\alpha}(1-\beta \cos \theta)^{-(2+\alpha)}$

where $\alpha$ is the jet spectral index $\left(\mathrm{S}(\nu) \propto \nu^{-\alpha}\right)$. Some problems can be related to this measurement: free free absorption may affect the observed jet brightness, moreover, we cannot derive strong constraints in intrinsically low luminosity jets: e.g. in 3C264 where a well studied optical and radio jet is present (15) the highest $\mathrm{j} / \mathrm{cj}$ ratio is $\mathrm{R}>37$ which implies that the source is oriented at $\theta<52^{\circ}$ with a jet moving with $\beta>0.62$.

\subsection{Radio Core Dominance}

The core radio emission measured at arcsecond resolution is dominated by the Doppler-boosted pc-scale relativistic jet. The source radio power measured at low frequency (e.g. 408 $\mathrm{MHz}$ ), instead, is due to the extended emission, which is not affected by Doppler boosting. At low frequency the observed core radio emission is not relevant since it is mostly selfabsorbed. Given the existence of a general correlation between the core and total radio power discussed in (11), we can derive the expected intrinsic core radio power from the unboosted total radio power. The comparison between the expected intrinsic core radio power and the observed core radio power will give constraints on the jet velocity and orientation (11). We note that the core radio power is best measured at $5 \mathrm{GHz}$ where it is dominant because of the steep spectrum of the extended emission, self-absorption is not relevant, and high angular resolution images allow us to separate the core from the extended jet emission.

Plotting the observed core radio power at $5 \mathrm{GHz}$ against the total radio power at $408 \mathrm{MHz}(9)$ we find 
a large dispersion in the core radio power. This is expected because of the strong dependance of the observed core radio power on $\theta$ and $\beta$. From the data dispersion, assuming that no selection effect is present in the source orientation $\left(\theta=0^{\circ}\right.$ to $\left.90^{\circ}\right)$, we can derive that the jet Lorentz factor $\gamma$ has to be $<10$ otherwise we should observe a larger core radio power dispersion.

A problem related with this measurement is a possible nuclear intrinsic variability. However, we note that a flux density variability larger than a factor 2 in the nuclear radio emission is uncommon.

\subsection{Other Methods}

Synchrotron-Self-Compton Emission: in principle when the core angular size is known, the comparison between the observed and predicted nuclear non thermal X-ray emission gives a limit to the source Doppler factor $\delta(8)$. A problem in this approach is the too large uncertainty in the observed nuclear X-Ray emission and in the core angular size.

Arm Length Ratio: by comparison of the size of the approaching $\left(\mathrm{L}_{a}\right)$ and receding jet $\left(\mathrm{L}_{r}\right)$ we derive:

$\mathrm{L}_{a} / \mathrm{L}_{r}=(1+\beta \cos \theta)(1-\beta \cos \theta)^{-1}$

A major problem is that the source size is related to the uv coverage and image sensitivity, moreover, the jet interaction with the ISM can affect the source size.

Brightness Temperature: estimates of the $\mathrm{T}_{b}$ from source variability or high resolution images (e.g. Space VLBI) can give constraints on the source Doppler factor (up to 1000 for Intra Day Variable (IDV) sources). I will not discuss this point, since IDV is still a hot and controversial topic (see (4)) .

Readhead (17) suggested an alternative method:

$\delta=\mathrm{T}_{b, V L B I} / T_{e q}$

where the brightness temperature under equipartition conditions is $\mathrm{T}_{e q}$ $\sim 510^{10} \mathrm{~K}$.

\section{Results}

In the literature many single sources and/or samples have been studied. In most cases observational data are in agreement with Unified Scheme Models and with the presence of a high velocity pc scale jet.

To derive statistical properties of radio jets on the pc scale, we extended the results discussed in (11) selecting all sources of the 3CR and B2 catalogues in a sky region, with $\mathrm{z}$ $<0.1$ (to avoid selection effects on the source orientation and core radio power).

The sample consists of 95 sources, 53 of which have been observed with VLBI. Among them we have 32 FR I, 10 FR II, 10 compact flat spectrum sources (2 BL-Lacs, 1 CSO), and 1 CSS. From these preliminary results (Giovannini et al., in preparation), we found that in all sources pc scale jets move at high velocity. No correlation has been found between the 


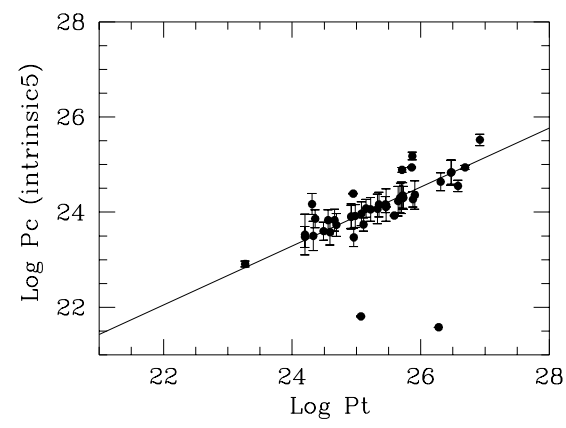

Fig. 1. Total radio power at $408 \mathrm{MHz}$ versus the intrinsic core radio power at 5 $\mathrm{GHz}$ derived with $\gamma=5$. The continuum line represents the general correlation between the core and the total radio power (see (11)).

jet velocity and the core or total radio power. Highly relativistic parsec scale jets are present regardless of the radio source power. Sources with a different kpc scale morphology, and total radio power have pc scale jets moving at similar velocities.

We used the estimated jet $\beta$ and $\theta$ to derive the Doppler factor $\delta$ for each source, and the corresponding intrinsic core radio power (assuming $\alpha=0)$ :

$\mathrm{P}_{c-\text { observed }}=\mathrm{P}_{c-\text { intrinsic }} \times \delta^{2}$

We found a good correlation between $\mathrm{P}_{c-\text { intrinsic }}$ and $\mathrm{P}_{t}$ with a small dispersion since plotting $\mathrm{P}_{c-\text { intrinsic }}$, we removed the spread due to the different orientation angles (Fig. 1). We found that a Lorentz factor $\gamma$ in the range 3 to 10 is consistent with the observational data. The Lorentz factor cannot be $>10$ for the previous considerations (Sect. 2.3). It cannot be $<3$ to remove the dispersion due to the different source orientations. The result that sources with different $\mathrm{P}_{t}$ and kpc scale morphology show the same correlation, implies that all pc scale jets have a similar velocity. Two sources do not follow the general correlation: M87 where we should have a higher jet velocity to fit with the general correlation and 3C 192 which core radio power is lower than expected (it could be in a pre-relic phase).

\section{Acceleration and Decelera- tion in Jets}

In some sources evidences of increasing velocity in pc scale jets have been found: e.g. M87 (2); 3C84 (6) Cygnus A (13). However, in these sources the jet velocity was measured from the jet apparent motion. Thus the increasing velocity could be non intrinsic but due to a change in the jet direction or to a change in the jet pattern velocity unrelated to the jet bulk velocity. In NGC 315 Cotton et al. (5) found an increasing jet velocity in the 5 inner pc from the core both from proper motion measurements AND from the sidedness ratio. A jet deceleration is evident in the FR I radio sources (see Laing this volume). In many low power sources there are observational evidences that jets are relativistics at their beginning and strongly decelerate within a few kpc from the core (see e.g. 3C449 (7)) .

In FR II radio sources kpc scale jets are still affected by Doppler boosting effects, but the jet sidedness ratio decreases with the core distance implying a velocity decrease. Observational data are consistent with $\gamma$ $\sim 2$ on the kpc scale (3). 


\section{$5 \quad$ Velocity Structures}

An evident limb-brightened jet morphology on the pc scale is present in some FR I sources as $1144+35$, Mkn 501, 3C264, M87, 0331+39 (11), and also in a few high power radio sources as 1055+018 (1), 3C 236 (Giovannini et al. in preparation). We interpret the limb-brightened structure as due to a different Doppler boosting effect in a two-velocity relativistic jet. If the source is oriented at a relatively large angle with respect to the line of sight, the inner very high velocity spine could be strongly deboosted, while the slower external layer could be boosted and become brighter than the inner jet region. Therefore only sources in a small range of $\theta$ will appear limb-brightened. For this reason, and for the observational difficulties of transversally resolving the radio jets, we expect that the number of sources exhibiting limb-brightened jets is low, as observed.

At present it is not clear if the velocity structure is strictly related to the jet interaction with the ISM as suggested by (11) or it is an intrinsic jet property. The presence of this structure very near to the central core as in M87 (14) and MKn 501 (Giroletti et al. in preparation) is in favour of a jet intrinsic property in the inner region (as discussed by Meier in this volume), whereas a jet velocity decrease due to the ISM is likely to be present in an intermediate region (from the pc to the kpc scale) (11).

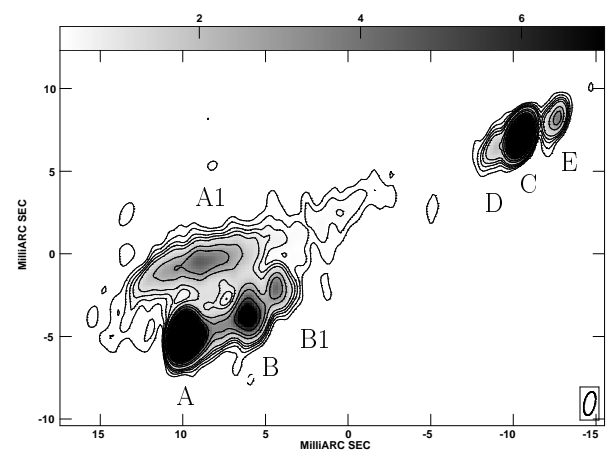

Fig. 2. VLBI Image of $1144+35$ at 8.4 GHz. Labels are: A,A1,B, and $\mathrm{B} 1$ jet substructures moving with $\beta_{a}=2.7 ; \mathrm{D}$ the jet beginning, $\mathrm{C}$ the core, $\mathrm{E}$ the counter-jet.

\section{The Source 1144+35}

$1144+35$ is a giant radio galaxy which shows a strong extended jet and a short counter-jet on the pc scale (10). The main jet is limb-brightened with evident substructures in the external shear layer which are moving with $\beta_{a} \sim 2.7$ (Fig. 2). New recent observations at $8.4 \mathrm{GHz}$ have allowed to measure a possible proper motion also in the cj: $\beta_{a-c j}=0.3 \pm 0.1$ in the time range 1995 - 2002 with 4 different epochs (Giovannini et al. in preparation). The jet bulk velocity is in the range $0.8 c-0.9 c$, as derived from the jet sidedness ratio, for the bright jet external regions. The pattern velocity, derived from the main jet proper motion in the same regions, is $>0.8 \mathrm{c}$. In both cases the derived jet orientation angle to the line of sight is $\theta<37^{\circ}$. Moreover, according to (16) using both the jet and counter jet proper motions we can obtain directly $\theta=30^{\circ}$ and $\beta=$ 0.9. From these data we can obtain a comprehensive scenario, as follows: 1) the bulk and pattern velocity are 
the same; 2) the shear layer Doppler factor is $\sim 2$ (being $\gamma \sim 2.3$ ) while assuming $\gamma=15$ for the inner spine, its Doppler factor is 0.7 in agreement with the observed brightness distribution; 3) if the external shear layer started with the same velocity of the inner spine, its velocity decreased from 0.998 c to 0.9 c in less than 100 pc suggesting an intrinsic origin for the jet velocity structure (Meier this volume).

\section{Conclusions}

The jet velocity and orientation can be estimated with good observational data. In a few sources the bulk and pattern jet velocity are the same, but the problem is still open.

The pc scale jet velocity is highly relativistic $(\gamma=3$ to 10) in both high and low power radio sources, with no relation with the total radio power and the large scale morphology.

The jet velocity from the pc to the kpc scale decreases dramaticaly in FR I radio sources and more slowly in FR II sources. In a few cases a jet acceleration has been found in the source inner regions (few pc).

A two velocity regime has been found in some low power sources and in a few high power sources. The different Doppler factor can explain the observed limb-brightened structures visible in some high resolution images. The presence of a shear layer could be an intrinsic property; a velocity decrease in the shear layer could be due to the interaction with the surrounding ISM in the tens of pc - kpc scale.

\section{References}

[1] Attridge J.M., et al. 1999 ApJ, 518, L87

[2] Biretta J.A., Zhou F., Owen F.N. 1995 ApJ, 447, 582

[3] Bridle A.H., et al. 1994 AJ, 108, 766

[4] Cimo' G., et al. 2002 in Proceedings of the 6th European VLBI Symp., E. Ros, et al. eds., p. 69

[5] Cotton W.D., et al. 1999 ApJ, 519, 108

[6] Dhawan V., Kellerman K.I., Rommney J.D. 1998 ApJ, 498, 111

[7] Feretti L. et al. 1999 A\&A 341, 29

[8] Ghisellini G., et al. 1993, ApJ, 407, 65

[9] Giovannini G., et al. 1988 A\&A, 199, 73

[10] Giovannini G., et al. 1999 ApJ, 522,101

[11] Giovannini G., et al. 2001 ApJ, 552,508

[12] Kellerman K.I., et al. 2000 in Astrophys. Fenomena Revealed by Space VLBI, H. Hirabayashi, et al. eds, Japan, p. 19

[13] Krichbaum T.P., et al. 1998 A\&A 329, 873

[14] Junor W., Biretta J.A., Livio M. 1999 Nature, 401, 891

[15] Lara L., et al. 1999 ApJ, 513, 197

[16] Mirabel I.F., \& Rodriguez, L.F. 1994 Nature, 371, 46

[17] Readhead A.C.S. 1994 ApJ, 426, 51

[18] Urry C.M. \& Padovani P. 1995 PASP, 107, 803

[19] Vermeulen R.C., \& Cohen M.H. 1994 ApJ, 430, 467 